\title{
THE TOPOLOGICAL STRUCTURE OF THE SPACE OF ALGEBRAIC VARIETIES
}

\author{
H. BLAINE LAWSON, JR.
}

The intention of this note is to announce some recent results concerning the homotopy type of the Chow variety of projective algebraic varieties. We begin by discussing the case of complex projective $n$-space $\mathbf{P}^{n}$.

For fixed integers $d \geq 1$ and $p, 0 \leq p<n$, let $C_{p, d}\left(\mathbf{P}^{n}\right)$ denote the set of effective $p$-cycles of degree $d$ in $\mathbf{P}^{n}$. This is defined to be the family of all finite sums $c=\sum n_{\alpha} V_{\alpha}$, where for each $\alpha, n_{\alpha}$ is a positive integer and $V_{\alpha} \subset \mathbf{P}^{n}$ is an irreducible subvariety of dimension $p$, and where degree $(c)=\sum n_{\alpha}$ degree $\left(V_{\alpha}\right)=d$. The set $C_{p, d}\left(\mathbf{P}^{n}\right)$ itself carries the structure of a projective algebraic variety. In particular it has a natural topology.

The spaces $C_{p, d}\left(\mathbf{P}^{n}\right)$, for distinct values of $d$, are conventionally considered to be mutually disjoint. However, if we fix a "distinguished" $p$-dimensional linear subspace $l_{0} \subset \mathbf{P}^{n}$, we obtain a natural sequence of topological embeddings,

$$
\cdots \subset C_{p, d}\left(\mathbf{P}^{n}\right) \subset C_{p, d+1}\left(\mathbf{P}^{n}\right) \subset \cdots,
$$

given at each level by mapping $c$ to $c+l_{0}$. We can then consider the union of these spaces

$$
C_{p}\left(\mathbf{P}^{n}\right)=\lim _{d} C_{p, d}\left(\mathbf{P}^{n}\right)
$$

endowed with the weak limit topology. This is the topology in which a set $C$ is closed if and only if the intersections $C \cap C_{p, d}\left(\mathbf{P}^{n}\right)$ are closed for all $d$. Note that $C_{p}\left(\mathbf{P}^{n}\right)$ is an abelian topological semigroup with unit $\left[l_{0}\right]$. Our first result is the following.

THEOREM 1. For all $n$ and $p, 0 \leq p<n$, there is a homotopy equivalence

$$
C_{p}\left(\mathbf{P}^{n}\right) \stackrel{\text { h.e. }}{\sim} K(\mathbf{Z}, 2) \times K(\mathbf{Z}, 4) \times \cdots \times K(\mathbf{Z}, 2(n-p)),
$$

where $K(\mathbf{Z}, l)$ denotes the standard Eilenberg-Mac Lane space.

We recall that for an integer $l$ and a finitely generated abelian group $G$, the Eilenberg-Mac Lane space $K(G, l)$ is a countable CW complex uniquely defined up to homotopy type by the requirement that: $\pi_{l}(K(G, l)) \cong G$ and $\pi_{m}(K(G, l))=0$ for $m \neq l$. The space $K(G, l)$ classifies the functor $H^{l}(\cdot ; G)$, i.e., there is a natural isomorphism $H^{l}(X ; G) \cong[X, K(G, l)]$ for any finite complex $X$. Under this equivalence the cohomology of $K(G, l)$ is carried over

Received by the editors January 23, 1987 and, in revised form, June 8, 1987.

1980 Mathematics Subject Classification (1985 Revision). Primary 14C05; Secondary 55P15, 55Q52.

Research partially supported by NSF Grant No. DMS8602645 and the JSPS. 
to cohomology operations. For this reason the cohomology of $K(\mathbf{Z}, l)$ (and $\left.K\left(\mathbf{Z}_{m}, l\right)\right)$ was computed in detail in the 1950 s.

There are two special cases of Theorem 1 which are worth noting.

EXAMPLE. $(p=n-1)$. One can easily see that $C_{n-1}\left(\mathbf{P}^{n}\right) \cong \mathbf{P}^{\infty}$, i.e., infinite-dimensional complex projective space, written as a monotone union of finite-dimensional linear subspaces and given the weak topology. The homotopy equivalence $\mathbf{P}^{\infty} \sim K(\mathbf{Z}, 2)$ is elementary and well known.

EXAMPLE. $(p=0)$. The space $C_{0}\left(\mathbf{P}^{n}\right)$ corresponds to the infinite symmetric product as defined by Dold and Thom, and in this case Theorem 1 is just a special case of their classical results $[2, \mathbf{3}]$.

It should be remarked that the author was led to Theorem 1 in part by an old conjecture of $\mathrm{H}$. Federer, which was based on the work of Dold and Thom and was proved by F. Almgren in 1962 [1].

Theorem 1 has a substantial generalization which is formulated as follows. Fix a linear embedding $\mathbf{P}^{n} \subset \mathbf{P}^{n+k}(k \geq 1)$ and choose a linear subspace $\mathbf{P}^{k-1} \subset \mathbf{P}^{n+k}$ disjoint from $\mathbf{P}^{n}$. Projecting away from $\mathbf{P}^{k-1}$ and onto $\mathbf{P}^{n}$ makes $\mathbf{P}^{n+k}-\mathbf{P}^{k-1}$ into a holomorphic vector bundle

$$
\pi: \mathbf{P}^{n+k}-\mathbf{P}^{k-1} \rightarrow \mathbf{P}^{n}
$$

of rank $k$ ( $k$ copies of $\mathcal{O}(1))$. Given any closed set $X \subset \mathbf{P}^{n}$ we define its complex $k$-fold suspension to be the set

$$
\not^{k} X=\overline{\pi^{-1}(X)}
$$

Note that $\not^{k} X$ is just the union of all lines joining $X$ to $\mathbf{P}^{k-1}$, i.e., the "complex join" $X \nVdash_{\mathbf{C}} \mathbf{P}^{k-1}$. When $k=1, \not \subset X$ is the Thom space of the hyperplane bundle $O(1)$ restricted to $X$. In general, $\not^{k}(X)=\not 4(\not 4(\ldots(X) \ldots))$ is obtained by iterating this Thom construction.

If $X \subset \mathbf{P}^{n}$ is an algebraic variety, so is $\not^{k} X$. In fact $\not^{k} X$ is defined by the same polynomials considered now to have $k$ additional "secret" variables. The map of algebraic sets $V \rightarrow \not^{k} V$ extends naturally to algebraic cycles. In fact, if $l_{0}^{p} \subset \mathbf{P}^{n}$ is our distinguished $p$-dimensional linear subspace, we set $l_{0}^{p+k}=\not^{k} l_{0}^{p}$ and obtain a map

$$
\not^{k}: C_{p}\left(\mathbf{P}^{n}\right) \rightarrow C_{p+k}\left(\mathbf{P}^{n+k}\right) .
$$

Suppose now that $X \subset \mathbf{P}^{n}$ is a closed subset which contains $l_{0}$, and define

$$
C_{p}(X)^{\wedge}=\left\{c \in C_{p}\left(\mathbf{P}^{n}\right): c \subset X\right\} .
$$

By " $c \subset X$ " we mean that $c$ can be written as $c=\sum n_{\alpha} V_{\alpha}$ where $V_{\alpha} \subset X$ for all $\alpha$. Let $C_{p}(X)$ denote the connected component of $C_{p}(X)^{\wedge}$ containing $l_{0}$.

THEOREM 2. For any algebraic subvariety $X \subset \mathbf{P}^{n}$ with $l_{0} \subset X$, and for all $k \geq 1$, the embedding

$$
\not^{k}: C_{p}(X) \rightarrow C_{p+k}\left(\not \not^{k} X\right)
$$

is a homotopy equivalence.

Specializing to the case where $p=0$ and $X=\mathbf{P}^{n}$ and applying the result of Dold and Thom, we recapture Theorem 1 from Theorem 2. 
The assumption that $l_{0} \subset X$ is always fulfilled when $p=0$. Hence, for every variety $X$ and every integer $k \geq 0$ there is a homotopy equivalence

$$
C_{0}(X) \stackrel{\text { h.e. }}{\rightarrow} C_{k}\left(\not^{k} X\right) \text {. }
$$

From the theorem of Dold and Thom we immediately conclude the following:

COROLlARY 3. For any connected algebraic variety $X \subset \mathbf{P}^{n}$ there exist natural isomorphisms

$$
\pi_{*}\left(C_{k}\left(\not \not^{k} X\right)\right) \stackrel{\approx}{\rightarrow} H_{*}(X ; Z)
$$

for all $k \geq 0$.

Analogous results hold for the space of cycles reduced $\bmod m$. Let $X \subset \mathbf{P}^{n}$ be a closed subset with $l_{0} \subset X$, and let $m$ be a positive integer. We denote by $C_{p}(X) \otimes \mathbf{Z}_{m}$ the group of algebraic $p$-cycles in $X$ with $\mathbf{Z}_{m}(=\mathbf{Z} / m \mathbf{Z})$ coefficients, modulo the cyclic subgroup $\mathbf{Z}_{m} \cdot l_{0} \cdot C_{p}(X) \otimes \mathbf{Z}_{m}$ carries a natural topology as the quotient of topological groups $G_{p}(X) / m G_{p}(X)$ where $G_{p}(X) \supset C_{p}(X)$ is the group extending $C_{p}(X)$ and defined by considering cycles with arbitrary integer coefficients. This topology is also defined by the flat norm on $C_{p}(X) \otimes \mathbf{Z}_{m}$ considered as a subset of the integral $2 p$-currents modulo $m$. (See $[4,4.2]$.) The definition given above extends to this case and we have the following result.

THEOREM 4. For any algebraic subvariety $X \subset \mathbf{P}^{n}$ with $l_{0} \subset X$ and for all integers $k \geq 0$ and $m \geq 2$, the embedding

$$
\not^{k}: C_{p}(X) \otimes \mathbf{Z}_{m} \rightarrow C_{p+k}\left(\not \not^{k} X\right) \otimes \mathbf{Z}_{m}
$$

is a homotopy equivalence.

COROLlARY 5. For any connected algebraic variety $X \subset \mathbf{P}^{n}$ there are natural isomorphisms

$$
\pi_{*}\left(C_{k}\left(\not^{k} X\right) \otimes \mathbf{Z}_{m}\right) \stackrel{\approx}{\rightarrow} H_{*}\left(X ; \mathbf{Z}_{m}\right)
$$

for all $k \geq 0$ and $m \geq 2$.

COROLlARY 6. For each $n$ and $p, 0 \leq p<n$, and for each $m \geq 2$, there is a homotopy equivalence

$$
C_{p}\left(\mathbf{P}^{n}\right) \otimes \mathbf{Z}_{m} \stackrel{\text { h.e. }}{\sim} K\left(\mathbf{Z}_{m}, 2\right) \times K\left(\mathbf{Z}_{m}, 4\right) \times \cdots \times K\left(\mathbf{Z}_{m}, 2(n-p)\right) .
$$

Suppose now that $X$ and $Y$ are subvarieties of $\mathbf{P}^{n}$ with $l_{0} \subset Y \subset X$. Every algebraic $p$-cycle $c \subset X$ can be written uniquely in the form $c^{\prime}+c^{\prime \prime}$ where $c^{\prime} \subset Y$ and where $c^{\prime \prime}$ has no component contained in $Y$. Two such cycles $c_{1}, c_{2} \subset X$ are said to be equivalent $(\bmod Y)$ if $c_{1}^{\prime \prime}=c_{2}^{\prime \prime}$. The set of equivalence classes is denoted $C_{p}(X, Y)$. Note that $C_{p}(X, Y)=C_{p}(X) / C_{p}(Y)$, i.e., $C_{p}(X, Y)$ is the space of maximal orbits of $C_{p}(Y)$ acting by translations on $C_{p}(X)$. 
THEOREM 7. For any pair of algebraic subvarieties $X, Y \subset \mathbf{P}^{n}$ with $l_{0} \subset$ $Y \subset X$ and for all integers $k \geq 0$, the map

$$
\not^{k}: C_{p}(X, Y) \rightarrow C_{p+k}\left(\not^{k} X, \not^{k} Y\right)
$$

is a homotopy equivalence.

The analogous statement is true for p-cycles with $\mathbf{Z}_{m}$-coefficients.

COROLlaRY 8. Let $X$ and $Y$ be connected varieties as in Theorem 7. Then there exist natural isomorphisms

$$
\begin{gathered}
\pi_{*}\left(\mathcal{C}_{k}\left(\not^{k} X, \not^{k} Y\right)\right) \rightarrow H_{*}(X, Y ; \mathbf{Z}), \\
\pi_{*}\left(C_{k}\left(\not^{k} X, \not^{k} Y\right) \otimes \mathbf{Z}_{m}\right) \rightarrow H_{*}\left(X, Y ; \mathbf{Z}_{m}\right),
\end{gathered}
$$

for all $k \geq 0$ and $m \geq 2$.

REMARK 9. From the nature of the proofs it seems possible that the results above could carry over to projective varieties defined over more general fields.

REMARK 10. It seems likely that there exists a sequence of integers $N_{d} \rightarrow$ $\infty$, depending on $p$ and $n$, such that the inclusion $C_{p, d}\left(\mathbf{P}^{n}\right) \rightarrow C_{p}\left(\mathbf{P}^{n}\right)$ is $N_{d}$-connected. There does, in fact, exist such a sequence so that the inclusion has a right homotopy inverse through this range. Hence Theorem 1 applies to give results about the connected components of the classical Chow variety.

SKETCH OF THE PROOFS. We consider only the basic case where $\not 4: C_{p}\left(\mathbf{P}^{n}\right) \rightarrow C_{p+1}\left(\mathbf{P}^{n+1}\right)$. We introduce an intermediate space $I \equiv\{c \in$ $C_{p+1}\left(\mathbf{P}^{n+1}\right): c$ has no component contained in $\left.\mathbf{P}^{n}\right\}$. The argument breaks into two parts.

Step 1. The inclusion $\not 4: C_{p}\left(\mathbf{P}^{n}\right) \rightarrow I$ is a homotopy equivalence. We show in fact that $C_{p}\left(\mathbf{P}^{n}\right)$ is a deformation retract of $I$. The deformation is given by the action of the linear flow which pulls away from $\mathbf{P}^{n}$ and toward the polar point $x_{0}=\mathbf{P}^{0}$.

Step 2. The inclusion $i: I \subset C_{p+1}\left(\mathbf{P}^{n+1}\right)$ is a homotopy equivalence. We show here that the map $i_{*}: \pi_{m} I \rightarrow \pi_{m} C_{p+1}\left(\mathbf{P}^{n+1}\right)$ is an isomorphism for all $m \geq 0$. This is the more subtle part of the argument, the part which requires passing to high degrees. Let us consider the question of the surjectivity of $i_{*}$. Suppose $f: S^{m} \rightarrow C_{p+1}\left(\mathbf{P}^{n+1}\right)$ is a continuous map (in fact a PL map for some triangulation of $\left.C_{p+1}\left(\mathbf{P}^{n+1}\right)\right)$. We show that there is an integer $d_{f}$ such that for all $d \geq d_{f}$ the map $d f=f+\cdots+f$ ( $d$ times) is homotopic to a map $\tilde{f}: S^{m} \rightarrow I$. (This will clearly prove the surjectivity of $i_{*}$.) The argument goes as follows. Fix a linear embedding $\mathbf{P}^{n+1} \subset \mathbf{P}^{n+2}$ and a point $x_{\infty} \in$ $\mathbf{P}^{n+2}-\mathbf{P}^{n+1}$. The projection $\pi: \mathbf{P}^{n+2}-\left\{x_{\infty}\right\} \rightarrow \mathbf{P}^{n+1}$ makes $\mathbf{P}^{n+2}-\left\{x_{\infty}\right\}$ into a complex line bundle.

Fix $d \in \mathbf{Z}^{+}$and let $\operatorname{Div}_{d}^{\prime}$ denote the set of divisors of degree $d$ on $\mathbf{P}^{n+2}$ which do not meet $x_{\infty}$. Given $D \in \operatorname{Div}_{d}^{\prime}$ and any $c \in C_{p+1}\left(\mathbf{P}^{n+1}\right)$ we can define a lifting of the cycle $d \cdot c$ to $D$ by setting

$$
\phi_{D}(d \cdot c)=\pi^{-1}(c) \cap D .
$$

Scalar multiplication by $t$ in the line bundle $\mathbf{P}^{n+2}-\left\{x_{\infty}\right\}$ transforms $D$ to a new divisor $t D$, and $\lim _{t \rightarrow 0} \phi_{t D}(d \cdot c)=d \cdot c$. 
We now choose a second point $x_{1} \in \overline{x_{0} x_{\infty}}$ and let $\pi_{1}: \mathbf{P}^{n+2}-\left\{x_{1}\right\} \rightarrow \mathbf{P}^{n+1}$ be the associated projection. If $x_{1} \notin t D$ for $0 \leq t \leq 1$ we can define a homotopy

$$
\Psi_{t}: d \cdot C_{p+1}\left(\mathbf{P}^{n+1}\right) \rightarrow C_{p+1}\left(\mathbf{P}^{n+1}\right)
$$

by setting

$$
\Psi_{t}=\pi_{1} \circ \phi_{t D}
$$

The map $\Psi_{0}$ is the identity on $d \cdot C_{p+1}\left(\mathbf{P}^{n+1}\right)$. Furthermore, for any given cycle $c$, we will have $\Psi_{t}(c) \subset I$ provided $c$ has no component which lies in the divisor $\alpha(t D)=\pi\left((t D) \cap \pi_{1}^{-1}\left(\mathbf{P}^{n}\right)\right) \subset \mathbf{P}^{n+1}$. It is elementary to show that given $f: S^{m} \rightarrow C_{p+1}\left(\mathbf{P}^{n+1}\right)$ there exists $D \in \operatorname{Div}_{d}^{\prime}$ so that $f(x)$ has no component in $\alpha(t D)$ for any $x \in S^{m}$ and any $t, 0 \leq t \leq 1$. It follows that $\Psi_{t}\left(d f\left(S^{m}\right)\right) \subset I$ for $0<t \leq 1$, and surjectivity is proved.

The proof of the injectivity of $i_{*}$ is similar.

The general case follows from the same argument given for the specific one.

The proof of Theorem 4 comes down to showing that the map $C_{p}(X) \rightarrow$ $C_{p}(X) / m \cdot C_{p}(X)=C_{p}(X) \otimes \mathbf{Z}_{m}$ is a quasifibration. Full details will appear elsewhere.

\section{REFERENCES}

1. F. J. Almgren, Jr., The homotopy groups of the integral cycle groups, Topology 1 (1962), 257-299.

2. A. Dold and R. Thom, Une généralisation de la notion d'espace fibré: Application aux produits symétriques infinis, C. R. Acad. Sci. Paris 242 (1956), 1680-1682.

3. __ Quasifaserungen und unendliche symmetrische produkte, Ann. of Math. 67 (1958), 239-281.

4. H. Federer, Geometric measure theory, Springer-Verlag, New York, 1969.

Department of Mathematics, State University of New York, Stony BROOK, NEW YORK 11794 